\title{
Laboreal
}

Volume $7 \mathrm{~N}^{\circ} 1$ | 2011

Psicodinâmica e psicopatologia do trabalho

\section{Higiene Pública : estadística de accidentes de trabajo}

Higiene Pública : estatística de acidentes de trabalho

Hygiène Publique : statistique d'accidents de travail

Public health : work accidents' statistics

\section{Armand Imbert y Antonim Mestre}

\section{(2) OpenEdition}

Journals

Edición electrónica

URL: http://journals.openedition.org/laboreal/8473

DOI: 10.4000/laboreal.8473

ISSN: 1646-5237

Editor

Universidade do Porto

Referencia electrónica

Armand Imbert y Antonim Mestre, «Higiene Pública : estadística de accidentes de trabajo », Laboreal [En línea], Volume $7 \mathrm{~N}^{0} 1$ | 2011, Publicado el 01 julio 2012, consultado el 24 septiembre 2020. URL : http://journals.openedition.org/laboreal/8473 ; DOI : https://doi.org/10.4000/laboreal.8473

Este documento fue generado automáticamente el 24 septiembre 2020

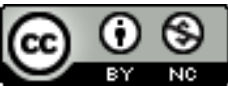

Laboreal está licenciado com uma Licença Creative Commons - Atribuição-NãoComercial 4.0 Internacional. 


\title{
Higiene Pública : estadística de accidentes de trabajo
}

\author{
Higiene Pública : estatística de acidentes de trabalho \\ Hygiène Publique : statistique d'accidents de travail \\ Public health : work accidents' statistics
}

Armand Imbert y Antonim Mestre

\section{REFERENCIA}

Artigo original : Imbert, A., \& Mestre, A. (1904). Hygiène Publique : statistique d'accidents de travail. Revue Scientifique, $\mathrm{n}^{\circ} 13$, Tome11 (p. 385-390).

1 Más allá de los medios científicos, cabe señalar, que los métodos y dispositivos de laboratorio pueden ayudar a resolver algunos de los problemas más graves existentes entre el capital y el trabajo. Aunque esto parezca una verdad evidente para los que se preocupan por este modo de uso práctico de los datos de la ciencia experimental, los propios interesados - jefes y trabajadores - no sospechan su existencia ; y los esfuerzos realizados para su divulgación constituyen, creemos, una obra esencialmente útil y provechosa, de interés general.

2 Dentro de los elementos que caracterizan en el problema social actual, cuya solución imparcial se puede buscar fuera de las discusiones apasionadas de las personas interesadas, se encuentran en particular la duración de la jornada (de trabajo) y, más genéricamente, la organización del trabajo [1].

3 Si la producción del trabajo resulta necesaria para asegurar y conservar el buen funcionamiento del motor animado que somos todos, si no se desea el deterioro del organismo, es necesario que el suministro de energía que debemos satisfacer no sobrepase determinados límites ; además, es peligroso teniendo en cuenta el desarrollo completo del organismo y su futuro rendimiento, que se haga un uso excesivamente 
precoz e intensivo. No es raro que para un mismo número de horas de trabajo existan interrupciones consecutivas de uno o varios períodos de descanso.

Los efectos nocivos de las prácticas que podrían ser condenables se pueden investigar a través de diversos métodos, algunos de los cuales exigen poner en práctica técnicas de laboratorio mientras que otros son simples interpretaciones de constataciones realizadas frecuentemente con otro propósito.

Dentro de estas últimas debemos incluir el estudio de las estadísticas de accidentes de trabajo declarados de forma oficial de acuerdo a la aplicación de la Ley del 9 de Abril de 1898 [2]. El hecho de consistir, al menos aparentemente, en un simple agrupamiento de números, no significa que el método no sea verdaderamente científico o, más rigurosamente, fisiológico de base.

6 Efectivamente, la fatiga como consecuencia del trabajo, que a su vez se traduce, en particular, en modificaciones derivadas del modo de funcionamiento del motor que trabaja: el músculo, y que consiste en una relajación y una disminución de la intensidad de la contracción. Sin entrar en detalles en los fenómenos que se manifiestan en el trabajador en rápidas sucesiones a partir del momento en que se le advierte del surgimiento de un evento súbito hasta que realiza movimientos de defensa o abandono destinados a alejarlo del peligro que amenaza, basta con decir que entre más cansado esté un trabajador, menos apto y preparado estará para realizar esos movimientos con la rapidez y la energía necesarias. En consecuencia, el número de accidentes debe aumentar con la fatiga de los trabajadores, y la distribución de estos accidentes de acuerdo a la hora de la jornada laboral en que se producen debe ser un indicador de evaluación del grado de fatiga de los trabajadores que los sufrieron.

7 Tal método de observación resulta, sin dudas, indirecto, y tales estadísticas presentan algunas incongruencias; en contrapartida, la distribución de los accidentes de acuerdo a las horas en que sucedieron tiene la ventaja de suministrar informaciones relacionadas con el conjunto de trabajadores. Las características individuales, el nivel de entrenamiento más o menos perfecto, la mayor o menor resistencia, etc.; desaparecen, y los resultados representan una medida de la cual se deducirán las indicaciones precisas sobre los cambios que se pueden introducir en la organización del trabajo de cada profesión.

8 Estas estadísticas pueden haber sido establecidas en el extranjero: al menos encontramos algunos "datos" en diversos trabajos sin conseguir aún encontrar estadísticas propias. Por el contrario, no parece que en Francia exista una distribución de los accidentes de acuerdo con el horario laboral y las ideas que están relacionadas con esta forma de clasificación no parecen comunes entre los principales interesados : trabajadores, jefes y compañías de seguros. En consecuencia, se puede determinar el interés que representa el estudio de los datos numéricos como resultado de la aplicación de la Ley del 9 de Abril de 1898 sobre los accidentes laborales.

Con esta Ley, entre otras prescripciones, se vuelve obligatorio al menos para ciertas profesiones la declaración de cualquier accidente que cause una incapacidad al trabajador de más de cuatro días, declaraciones que están centralizadas por zonas y por un funcionario o inspector del trabajo. Estos documentos oficiales fueron utilizados en primer lugar por el Departamento del Hérault que reúne un total de 56458 trabajadores, de diversas profesiones dentro de esta Ley, que fueron en su conjunto víctimas de 2065 accidentes declarados. 
La Figura 63 (curva con la línea continua) representa la distribución de acuerdo con la hora en que acontecieron los 660 accidentes relacionados a las profesiones calificadas oficialmente dentro de Mantenimiento y Transportes que engloban a 16695 trabajadores. Como consecuencia inmediata del análisis de esta curva podemos deducir lo siguiente :

$111^{\circ}$ - El número de accidentes aumenta progresivamente de hora en hora durante la primera mitad de la jornada laboral,

$122^{\mathrm{o}}$ - Después del descanso bastante prolongado del mediodía, en las primeras horas de la segunda mitad de la jornada laboral, el número de accidentes es significativamente menor que en la última hora de la mañana,

$133^{\text {o }}$ - En el transcurso de la segunda mitad de la jornada laboral, los accidentes se vuelven de nuevo más numerosos de forma progresiva,

$14 \quad 4^{0}$ - El número máximo de accidentes por hora cerca del fin de la segunda mitad de la jornada laboral es significativamente más elevado que su correspondiente máximo de la mañana.

15 No se podría, por lo visto, desear a priori una confirmación más rigurosa de las deducciones extraídas de la influencia de la fatiga de los trabajadores sobre la aparición de accidentes. De la misma forma, se debe concluir en primer lugar que los accidentes ocasionados por el ejercicio de otras profesiones traen como consecuencia los mismos resultados.

Figura 63 - Industria química (líneas discontinuas) - 1453 trabajadores, 326 accidentes ; Industria maderera (línea por puntos) - 4528 trabajadores, 189 accidentes

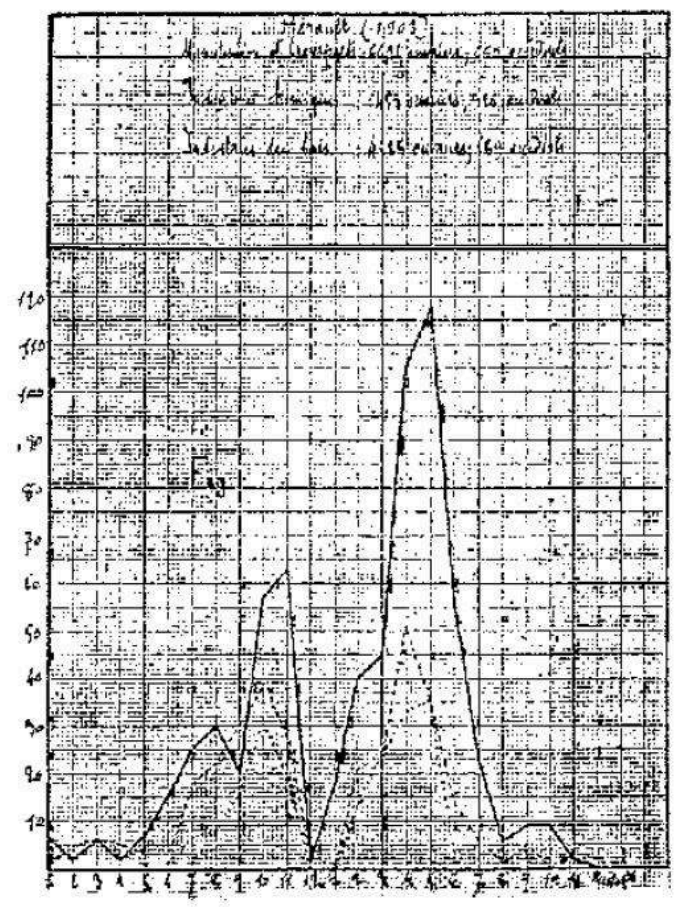

Figura 63

16 Esto es lo que muestran las curvas de líneas discontinuas y por puntos de la Figuras 63 y 64, representando la distribución por horas de los accidentes ocurridos en las siguientes profesiones : 
Figura 64 - Excavaciones y construcciones en piedra (línea continua) : 4686, 280 accidentes Trabajo con metales comunes (línea discontinua) : 8237 trabajadores, 149 accidentes Comercio y actividades bancarias (línea por puntos) : 15567 trabajadores, 237 accidentes

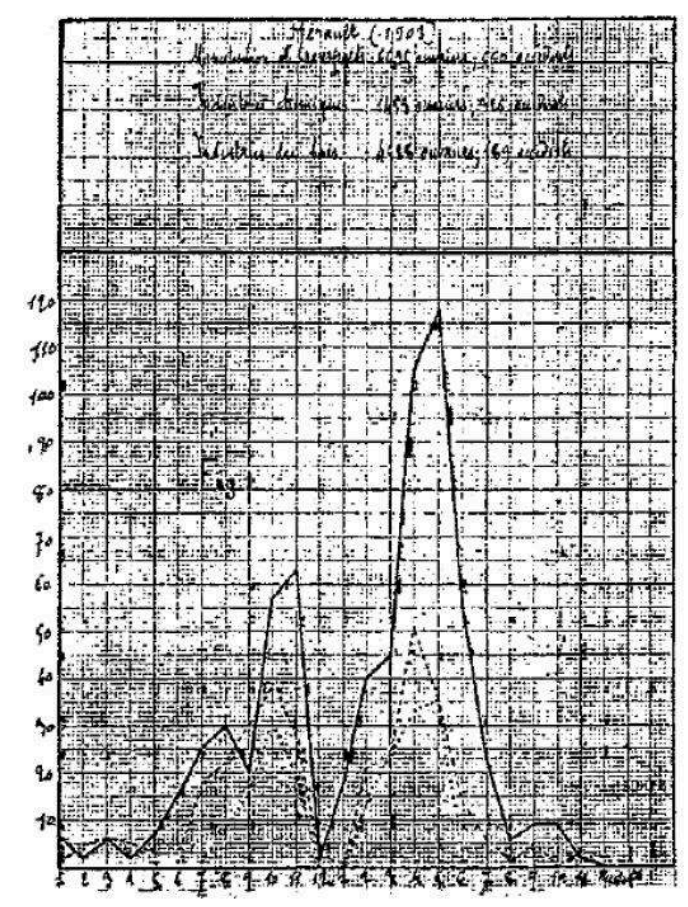

Figura 64

Comparando estas curvas unas con otras se notan algunas diferencias en cuanto a las horas en las cuales ocurre un máximo de accidentes. Tenemos motivos para pensar que estas diferencias se deben, por un lado, a que las estadísticas se refieren a un año entero, incluyendo verano e invierno, y, por tanto, las jornadas varían de duración con diferente distribución de los intervalos de descanso. Por otro lado, se debe también a que cada grupo engloba en la realidad a profesiones diferentes con horas de trabajo y descanso que no son exactamente las mismas.

El inspector de la División residente en Toulouse, el Señor Leroy, nos comunicó los resultados de estadísticas análogas, las que espontáneamente ofreció para que fuesen extraídas por los inspectores de los Departamentos bajos sus órdenes (Aude, Ariège, Aveyron, Cantal, Haute-Garonne, Lozère, Pirénées Ocidentales, Tarn-et-Garonne). Sin que reproduzcamos acá las curvas representativas de estas estadísticas diversas, podemos decir que todas, sin excepción alguna, presentan características generales como las que enunciamos anteriormente.

19 Estas mismas características se deben encontrar, entonces, en las curvas relativas al conjunto de todos los accidentes ocurridos en las diversas profesiones. Esto se muestra en la Figura 65, donde la línea continua representa, de acuerdo a la distribución de las horas, los 2065 accidentes de los que fueron víctima los 56458 trabajadores del Departamento de Hérault. La línea por puntos representa, en otra escala, la distribución por horas de los 5534 accidentes que ocurrieron con los 140467 trabajadores de los nueve Departamentos de la Circunscripción de Toulouse. A menos que serias críticas puedan ser reformuladas en contra de las estadísticas basadas en documentos oficiales, la distribución de los accidentes de acuerdo al horario laboral en que se producen 
muestra la influencia creciente de la fatiga del inicio al fin de cada media jornada de trabajo, con un máximo más acentuado en la tarde.

Las únicas críticas que podemos formular son las siguientes :

a. Debemos considerar - y sabemos que en algunas ocasiones así fue - que no todos los accidentes se declaran, lo que trae como consecuencia que ni las cifras citadas anteriormente, ni las curvas que deducimos de ellas corresponderían con la realidad. Sin embargo, se puede considerar de acuerdo con esto que las omisiones tal vez bastante numerosas al inicio de la aplicación de la Ley de 1898 deben ser ahora cada vez más raras. Ahora bien, nuestras estadísticas están recogidas en el año 1903 y así, las omisiones, si es que existen, no se pueden relacionar con una hora específica e invalidar de esta forma los resultados generales que se verificaron. Estos, salvo objeciones, se pueden considerar por tanto como un resultado con un grado de exactitud bastante aproximado a lo que se puede esperar en casos semejantes.

b. La crítica a continuación parece inicialmente la más grave: Si la fatiga es una causa de accidentes, el nivel en que se encuentra no lo es menos, y es generalmente proporcional a la gravedad de este, porque esta misma gravedad depende de circunstancias completamente fortuitas. Sin embargo, la Ley no exige otra cosa que la declaración de accidentes que sólo haya implicado una incapacitación del trabajo con una duración de al menos 4 días. Por lo tanto un buen número de accidentes que podrían tener una relación con la fatiga escapan a una declaración legal y no están incluidos en nuestras estadísticas, las cuales estarían falseadas.

21 En relación a esto, se puede considerar lo siguiente :

22 Aquellos accidentes ligeros que no se encuentran recopilados en una declaración legal y que no resultan de la fatiga deben (como en el caso de los accidentes no declarados y en función de las estadísticas de acuerdo a su relevancia) distribuirse de un modo aproximadamente uniforme en las diversas horas de la jornada laboral, lo que no altera la forma general de las curvas de las Figuras precedentes ni las consecuencias que fueron deducidas de estas Figuras. Por otro lado, si un cierto número de accidentes no declarados, debido a su gravedad leve, están ligados a pesar de todo a la fatiga, deberían ser más numerosos en las últimas horas de cada media jornada laboral y al introducirlo en las estadísticas resaltarían desde luego las características generales de las curvas de las Figuras 63, 64 y 65.

Se puede concluir, por tanto, que nuestras estadísticas de los accidentes relacionados con las horas de trabajo en las cuales estos acontecen muestran claramente la influencia que ejerce la fatiga profesional con nuestro modo de organización del trabajo sobre se trata de una ley con base al código del trabajo francés, el surgimiento de accidentes.

24 Hecha esta constatación, ¿qué podemos concluir ? Esto es debido a que, sobre todo en asuntos de esta índole, el valor de un hecho es tan importante cuanto mayores son las consecuencias prácticas que de se pueden obtener.

Con motivo de que la fatiga es una consecuencia inevitable de cualquier gasto de energía no se puede, por tanto, eliminarla sin eliminar al mismo tiempo a todo y a cualquier tipo de trabajo ; al menos sería necesario impedir que se alcanzase el grado a partir del cual su influencia en la aparición de los accidentes es nefasta. 
Figura 65

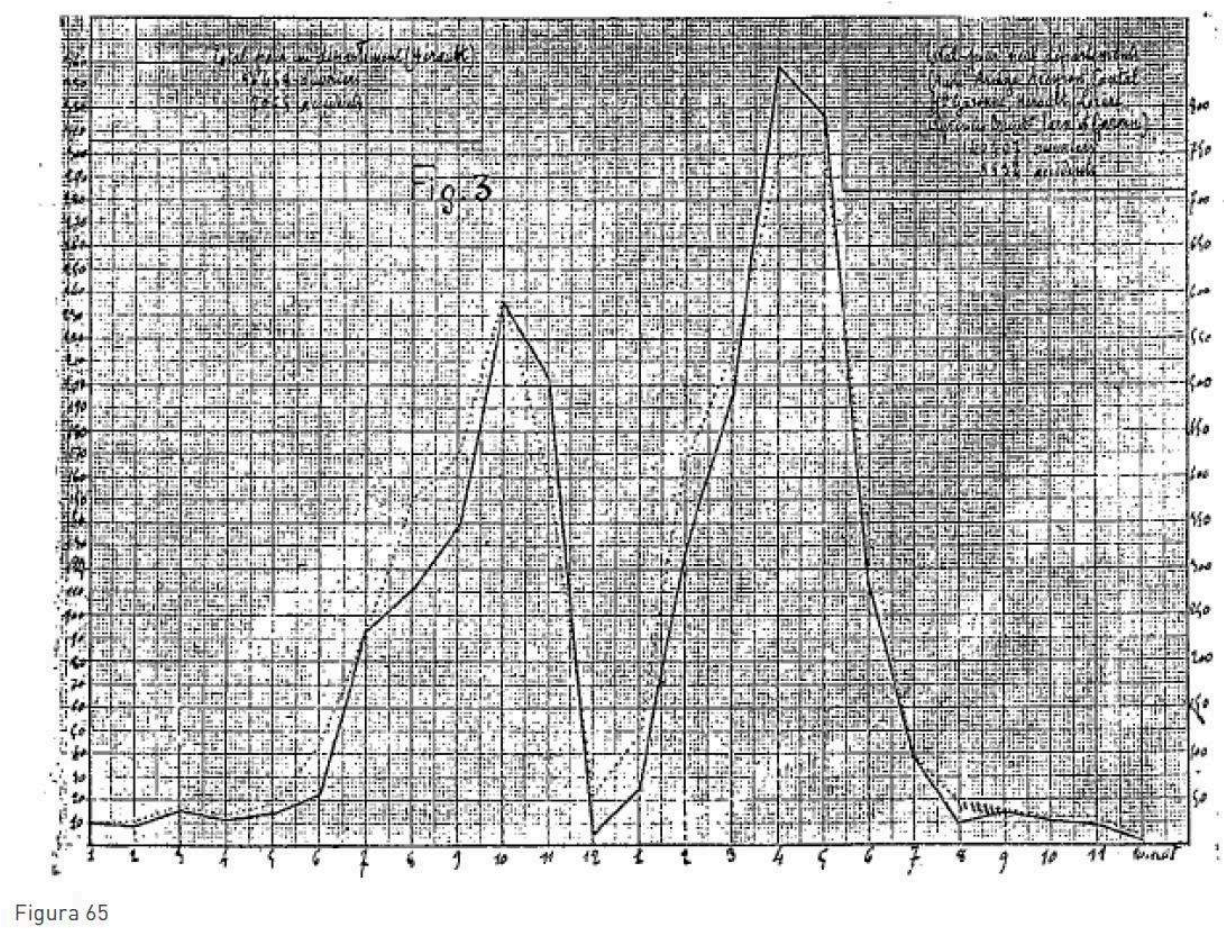

Si sólo tomamos en cuenta lo constatado durante las primeras horas en que se retoma el trabajo durante la tarde, parece suficiente para la determinación de una notoria disminución del número de accidentes el intercalar al medio de cada una de las dos partes de la jornada laboral un período de reposo, evidentemente menor que el del mediodía, porque la fatiga disminuiría, con una duración a determinar de acuerdo con las diversas consideraciones que se tienen que considerar en situaciones semejantes. Al editar tales prescripciones no se estaría haciendo sino aplicar al trabajo mecánico y a los adultos las medidas que hace mucho tiempo fueron puestas en práctica en niños con relación al trabajo mental.

Estadísticas semejantes a estas que dimos a conocer, pero con resultados de accidentes agrupados de acuerdo al día de la semana, muestran de la misma forma si el descanso del domingo es suficiente para que desaparezcan todos los vestigios de fatiga de la semana o si habría alguna justificación fisiológica para aumentar este descanso. Igualmente, nos dedicamos a establecer estadísticas semejantes en un cierto número de Departamentos del sur y daremos a conocer, posteriormente, las consecuencias derivadas. Al menos, podemos agregar por el momento que, habiendo encontrado en el Bulletin du Travail de Belgique accidentes agrupados por día de la semana, la comparación de los números no demuestra ningún aumento de lunes a sábado. Sin embargo, esto es un elemento que nos proponemos retomar en un futuro.

Los datos oficiales que son consecuencia de la aplicación de la Ley del 9 de Abril de 1898 permiten demonstrar otros elementos interesantes.

Cualquier declaración de accidentes lleva un resumen de las condiciones en las cuales ocurrió el accidente y a continuación la causa que lo produjo. Resulta fácil, de este modo, organizar los accidentes de acuerdo a las causas, lo que trae como consecuencia resultados que también se pueden representar gráficamente como hicimos en las 
Figuras 66, 67 y 68 para las profesiones calificadas oficialmente como : Mantenimiento y Transportes, Industria Maderera, Excavaciones y Construcciones en Piedra. Como resultado del examen de estas Figuras obtenemos para cada profesión una especie de accidente tipo, característico del género de trabajo propio de cada profesión $y$, por tanto, una causa de la mayor frecuencia de accidentes.

Figura 66

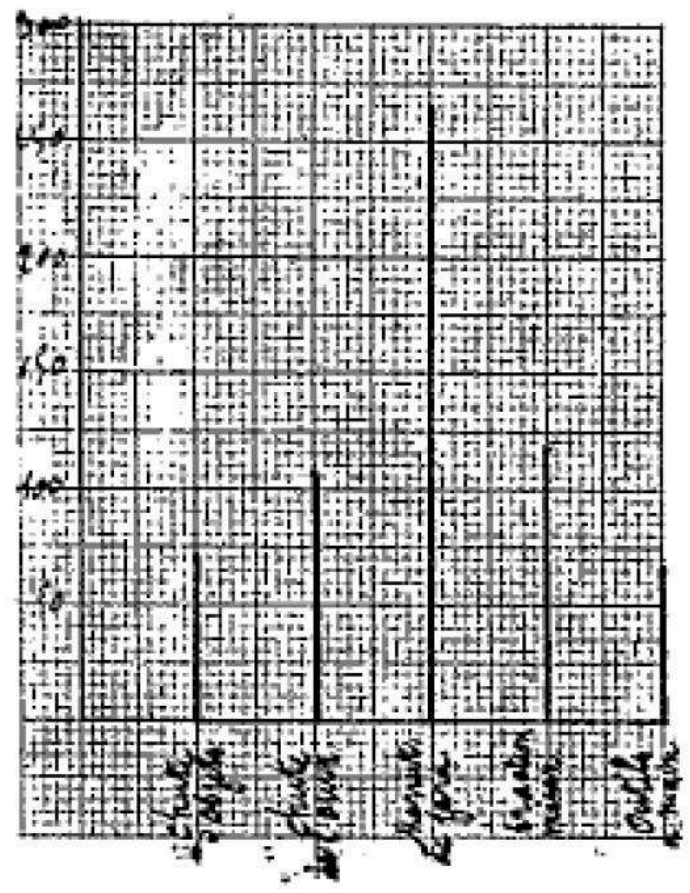

Figura 66

30 La importancia de esta idea es evidente. Si de hecho se pretende intentar reducir el número de accidentes eliminando otras causas además de la fatiga, podemos conocer para cada profesión cuál es el modo en que el esfuerzo y la vigilancia (en particular la del inspector del trabajo) deben incidir de forma más específica.

31 Además se puede, considerando la relación entre el número $N$ de trabajadores de una determinada profesión y el número $n$ de los que fueron víctimas de accidentes entre ellos, evaluar el riesgo medio que el ejercicio de esa profesión acarrea. A continuación presentamos el índice de los resultados proporcionados por el Departamento de Hérault para el cálculo de las relaciones que acabamos de definir.

\begin{tabular}{|l|l|}
\hline Profesiones & Relación N/n \\
\hline Mantenimiento y Transportes & 10,14 \\
\hline Industria química & 4,15 \\
\hline Industria Maderera & 23,95 \\
\hline Excavaciones y construcciones en piedra & 16,73 \\
\hline
\end{tabular}




\begin{tabular}{|l|l|}
\hline Comercio, sistema bancario & 236,45 \\
\hline Trabajo con metales comunes & 21,72 \\
\hline
\end{tabular}

Figura 67

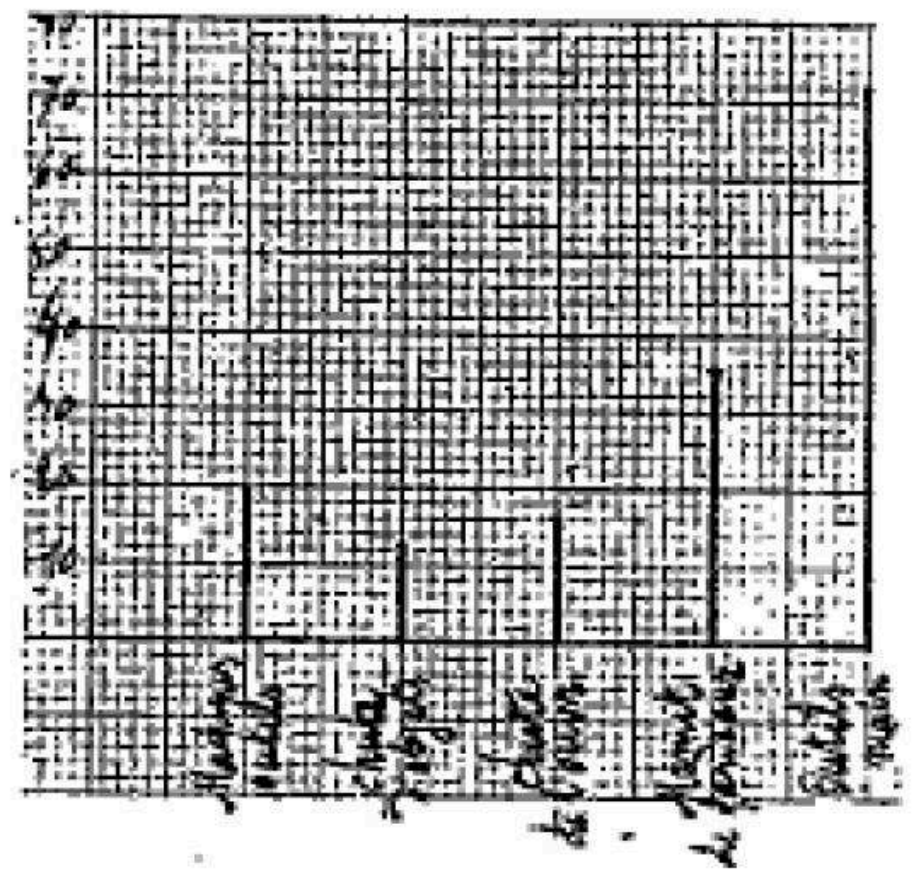

Figura 67

Las profesiones peligrosas son, por tanto, las que se encuentran en el apartado Industrias químicas, seguida inmediatamente de Mantenimiento y Transportes, etc. Existen, sin duda, alguna preocupaciones relacionadas con la exactitud absoluta de estos resultados: las Industrias químicas, por ejemplo, no emplearían en el Departamento de Hérault más de 1458 trabajadores, número que resulta relativamente poco elevado. Los valores numéricos de la relación $\mathrm{N} / \mathrm{n}$ de la tabla precedente no están, entonces, muy alejados de la realidad por lo que no se puede estar, sino intensamente, impresionado por las constataciones de que en cerca de cinco años en las Industrias químicas, y en diez $\mathrm{u}$ once años en el Mantenimiento $y$ Transportes, todos los trabajadores fueron sucesivamente víctimas de accidentes de acuerdo con el bis repetida... aunque esta sentencia no haya sido formulada para los accidentes de trabajo, un cierto número de estos ocurrieron en repetidas ocasiones.

33 Es, además, interesante hacer otra observación relacionada con la profesión de acuerdo a la importancia relativa a los accidentes tipo, importancia que se puede evaluar calculando la relación $n^{\prime}$ x 100/n, representando n' el número de accidentes tipo y $\mathrm{n}$ el número total de accidentes debido a cualquier causa.

Este cálculo conduce a los siguientes resultados

\begin{tabular}{|l|l|}
\hline Profesiones & n' $x 100 / \mathrm{n}$ \\
\hline
\end{tabular}




\begin{tabular}{|l|l|}
\hline Mantenimiento y Transportes & 39,7 \\
\hline Industria química & 22,1 \\
\hline Industria Maderera & 38 \\
\hline Excavaciones y construcciones en piedra & 29,2 \\
\hline Comercio, sistema bancario & 33,2 \\
\hline
\end{tabular}

Como resultado de estas cifras, obtenemos que las diversas profesiones presentan peligros y riesgos distintos como muestran los números de la Tabla precedente. La importancia del accidente tipo para cada una de estas profesiones oscila solamente entre $1 / 5$ y $1 / 3$ aproximadamente.

Terminando de esta forma nuestro estudio estadístico, no pensamos haber extraído de los datos oficiales resultantes de la aplicación de la Ley del 9 de Abril de 1898 todo lo que estos podrían aportar como nos proponemos comprobarlo posteriormente. Sólo pretendemos mostrar, con algunos ejemplos, cuán importantes son las ideas que se pueden extraer de los documentos oficiales relacionados con los accidentes de trabajo y que interés puede representar para los interesados, trabajadores y Compañías de Seguros, así como para los legisladores, la utilización de estas nociones con el fin de llevar a buen término un proyecto que todavía se encuentra lejos del objetivo que se pudiese proponer de llevar a cabo de forma razonable.

\section{NOTAS}

1. A. IMBERT. Les accidents du travail et les Compagnies d'assurances, (Revue Scientifique 4 juin 1901.)

2. se trata de una ley con base al código del trabajo francés

\section{AUTORES}

\section{ARMAND IMBERT}

Professor na Faculdade de Medicina de Montpellier

\section{ANTONIM MESTRE}

Inspector do trabalho no Departamento de Hérault 